\title{
KÄHLER DIFFERENTIAL MODULES AND CONFIGURATIONS OF POINTS IN $\mathbb{P}^{2}$
}

\author{
Tran Nguyen Khanh Linh ${ }^{\mathrm{a}^{*}}$, Le Ngoc Longa \\ ${ }^{a}$ Department of Mathematics, University of Education, Hue University, Thua Thien Hue, Vietnam \\ *Corresponding author: Email: tnkhanhlinh@hueuni.edu.vn
}

\section{Article history}

Received: May $7^{\text {th }}, 2021$

Received in revised form: May $24^{\text {th }}, 2021 \mid$ Accepted: July $26^{\text {th }}, 2021$

Available online: January $27^{\text {th }}, 2022$

\begin{abstract}
Given a finite set of points in the projective plane, we use the module of Kähler differentials to investigate the configurations of these points. More precisely, depending on the values of the Hilbert function of the module of Kähler differential 3-forms, we determine whether the set of points lies on a non-singular conic, or on two different lines, or on single line.
\end{abstract}

Keywords: Configuration of points; Finite set of points; Hilbert function; Kähler differential module.

DOI: http://dx.doi.org/10.37569/DalatUniversity.12.2.887(2022)

Article type: (peer-reviewed) Full-length research article

Copyright $(2022$ The author(s)

Licensing: This article is licensed under a CC BY-NC 4.0 


\section{INTRODUCTION}

The theory of Kähler differentials has been used very extensively in the literature as a tool to study finitely generated algebras. Many structural properties of the algebra can be reflected in terms of the modules of Kähler differentials, for instance, the smoothness criterion, the regular criterion, and the ramification criterion of the algebra (see Kunz, 1986; Scheja \& Storch, 1988). In de Dominicis and Kreuzer (1999), the authors introduced methods using this theory into the study of finite sets of points in the projective $n$-space $\mathbb{P}^{n}$ over a field $K$ of characteristic zero. Explicitly, given a set of points $\mathbb{X} \subseteq \mathbb{P}^{n}$ with homogeneous coordinate ring $R=K\left[X_{0}, \ldots, X_{n}\right] / I_{\mathbb{X}}$, the module of Kähler differential 1-forms of $R / K$ is $\Omega_{R / K}^{1}=\mathscr{J} / \mathscr{J}^{2}$, where $\mathscr{J}$ is the kernel of the multiplication map $\mu: R \otimes_{K} R \rightarrow R, f \otimes g \mapsto f g$. One of the main results of de Dominicis and Kreuzer (1999) is the formula

$$
\mathrm{HF}_{\Omega_{R / K}^{1}}(i)=(n+1) \mathrm{HF}_{\mathbb{X}}(i-1)-\sum_{j=1}^{n} \operatorname{HF}_{\mathbb{X}}\left(i-d_{j}\right)
$$

where $\mathbb{X}$ is a complete intersection of hypersurfaces of degrees $d_{1}, \ldots, d_{n}$. Also, they showed that the module $\Omega_{R / K}^{1}$ contains additional information about $\mathbb{X}$. Later, in Kreuzer et al. $(2015,2019)$, these differential algebra techniques were developed for arbitrary 0dimensional schemes in $\mathbb{P}^{n}$.

In this paper we apply the theory of Kähler differentials to investigate the geometrical structure of finite sets of points in the projective spaces. More precisely, we first look at the Hilbert functions of the modules of Kähler differential $m$-forms for a finite set of points in the projective $n$-space $\mathbb{P}^{n}$ over a field $K$ of characteristic zero and use these to determine the smallest projective space that contains the set of points. Then we restrict our attention to finite sets of points in $\mathbb{P}^{2}$ and show whether a set of points lies on a nonsingular conic, on two different lines, or on a line by detecting the Hilbert functions of the modules of Kähler differential 3-forms.

Now let us describe the content of the paper in detail. In Section 2, we first introduce the modules of Kähler differential $m$-forms and their basic properties for a finite set $\mathbb{X}$ of points in $\mathbb{P}^{n}$. In particular, we define the matrix of coordinates of points of $\mathbb{X}$ and show that the rank of the matrix is exactly the smallest positive integer $m$ such that the module of Kähler differential $m$-forms is not zero (see Theorem 2.6). Also, we show that the Hilbert function of the module of Kähler differential $m$-forms for a subset of $\mathbb{X}$ is bounded by that one for $\mathbb{X}$.

Next, in Section 3, we use the modules of Kähler differential $m$-forms for $\mathbb{X}$ to investigate the configurations of $\mathbb{X}$ in the case of $\mathbb{X} \subseteq \mathbb{P}^{2}$. Basically, we apply the Hilbert function of the module of Kähler differential 3-forms to characterize the geometrical properties of $\mathbb{X}$. Several nice properties about the shapes of $\mathbb{X}$ are provided. For example, Proposition 3.2 shows that $\mathbb{X}$ lies on a conic, not a double line, if and only if $\mathrm{HF}_{\Omega_{R / K}^{3}}(3)=1$ and $\mathrm{HF}_{\Omega_{R / K}^{3}}(i) \leq 1$ for all $i \geq 0$. In particular, Theorem 3.8 shows us how to determine whether the set $\mathbb{X}$ is on a single line, on two different lines, or on a non- 
singular conic by checking certain values of the Hilbert functions of $\Omega_{R / K}^{3}$.

All examples in this paper were calculated by using the computer algebraic system ApCoCoA (see ApCoCoA Team, 2021). Some of the results in this paper were improved and developed from results in Tran (2015).

\section{KÄHLER DIFFERENTIAL MODULES FOR SETS OF POINTS}

In the following let $K$ be a field of characteristic zero, let $S=K\left[X_{0}, \ldots, X_{n}\right]$, and let $\mathbb{X}=\left\{p_{1}, \ldots, p_{s}\right\}$ be a set of $s$ distinct $K$-rational points in the projective $n$-space $\mathbb{P}^{n}$. We assume that no point of $\mathbb{X}$ lies on the hyperplane at infinity $\mathscr{Z}^{+}\left(X_{0}\right)$. This enables us to write $p_{j}=\left(1: p_{j 1}: \cdots: p_{j n}\right)$ with $p_{j 1}, \ldots, p_{j n} \in K$ for $j=1, \ldots, s$. Each point $p_{j} \in \mathbb{X}$ has its associated homogeneous prime ideal in $S$ given by

$$
\mathfrak{p}_{j}:=\left\langle p_{j 1} X_{0}-X_{1}, \ldots, p_{j n} X_{0}-X_{n}\right\rangle \subseteq S .
$$

Then the homogeneous vanishing ideal of $\mathbb{X}$ is $I_{\mathbb{X}}=\bigcap_{j=1}^{s} \mathfrak{p}_{j}$ and the homogeneous coordinate of $\mathbb{X}$ is $R=S / I_{\mathbb{X}}$. The ring $R$ is a Cohen-Macaulay ring of dimension 1 and is also a graded $K$-algebra (see, e.g., Kreuzer \& Robbiano, 2005, Section 6.3, or Tran, 2015, Section 2.4).

The ring $R^{e}:=R \otimes_{K} R$ is known as the enveloping algebra of the algebra $R / K$. We have the canonical multiplication map $\mu: R^{e} \rightarrow R$ with $\mu(f \otimes g)=f g$ for all $f, g \in R$. It is clearly true that this map is homogeneous of degree zero and its kernel $\mathscr{J}:=\operatorname{Ker}(\mu)$ is a homogeneous ideal of $R^{e}$. For a homogeneous system of generators of $\mathscr{J}$ (see Kunz, 2005, Theorem G.7), these notions lead us to the following definition of Kähler differential modules of the algebra $R / K$.

Definition 2.1. Let $m$ be a positive integer.

(a) The graded $R$-module $\Omega_{R / K}^{1}:=\mathscr{J} / \mathscr{J}^{2}$ is called the module of Kähler differentials of $R / K$. The homogeneous $K$-linear map $d: R \rightarrow \Omega_{R / K}^{1}$ given by $f \mapsto$ $f \otimes 1-1 \otimes f+\mathscr{J}^{2}$ is called the universal derivation of $R / K$.

(b) The exterior power $\Omega_{R / K}^{m}:=\bigwedge_{R}^{m} \Omega_{R / K}^{1}$ is called the module of Kähler differential m-forms of $R / K$.

We denote the image of $X_{i}$ in $R$ by $x_{i}$ for $i=0, \ldots, n$. Then we have $\operatorname{deg}\left(d x_{i}\right)=$ $\operatorname{deg}\left(x_{i}\right)=1$ and $\Omega_{R / K}^{1}=R d x_{0}+\cdots+R d x_{n}$. Hence, $\Omega_{R / K}^{m}=0$ if $m \geq n+2$, and for $m \geq 1$, $\Omega_{R / K}^{m}$ is a finitely generated graded $R$-module, and its Hilbert function is defined by

$$
\mathrm{HF}_{\Omega_{R / K}^{m}}(i):=\operatorname{dim}_{K}\left(\Omega_{R / K}^{m}\right)_{i} \text {, for all } i \in \mathbb{Z} \text {. }
$$

Moreover, from from Kunz (2005), Proposition 4.12, we get the following presentation for $\Omega_{R / K}^{m}$. 
Proposition 2.2. For $1 \leq m \leq n+1$, the graded $R$-module $\Omega_{R / K}^{m}$ has a presentation

$$
\Omega_{R / K}^{m} \cong \Omega_{S / K}^{m} /\left(I_{\mathbb{X}} \Omega_{S / K}^{m}+d I_{\mathbb{X}} \Omega_{S / K}^{m-1}\right)
$$

where $d I_{\mathbb{X}}=\left\langle d F \mid F \in I_{\mathbb{X}}\right\rangle$.

In view of Kunz (2005), Proposition 4.4, the Hilbert polynomial of the module of Kähler differential $m$-forms $\Omega_{R / K}^{m}$ can be described as follows.

Proposition 2.3. For $1 \leq m \leq n+1$, the Hilbert polynomial of $\Omega_{R / K}^{m}$ satisfies

$$
\mathrm{HP}_{\Omega_{R / K}^{m}}(z)= \begin{cases}s & \text { if } m=1 \\ 0 & \text { if } m \geq 2\end{cases}
$$

In addition, the regularity index of $\Omega_{R / K}^{m}$ is bounded by $\operatorname{ri}\left(\Omega_{R / K}^{m}\right) \leq 2 \operatorname{ri}(R)+m$.

The next result, found in Scheja and Storch (1988) Proposition 85.12 is useful for the proof of Theorem 2.6.

Lemma 2.4. For two R-modules $M$ and $N$, there is a canonical isomorphism

$$
\bigwedge_{R}^{n}(M \oplus N) \cong \sum_{i=0}^{n} \bigwedge_{R}^{n-i}(M) \otimes_{R} \bigwedge_{R}^{i}(N) .
$$

Lemma 2.5. Let $1 \leq m \leq n+1$ and let $\mathscr{K}$ be the graded $R$-module

$$
\mathscr{K}=\left\langle\left(\frac{\partial F}{\partial x_{0}}, \ldots, \frac{\partial F}{\partial x_{n}}\right) \in R^{n+1} \mid F \in I_{\mathbb{X}}\right\rangle .
$$

Then there is an exact sequence of graded $R$-modules

$$
0 \longrightarrow \mathscr{K} \wedge_{R} \bigwedge_{R}^{m}\left(R^{n+1}\right) \longrightarrow \bigwedge_{R}^{m+1}\left(R^{n+1}\right) \longrightarrow \Omega_{R / K}^{m+1}(m+1) \longrightarrow 0 .
$$

Proof.

By de Dominicis and Kreuzer (1999), Proposition 1.3, we have an epimorphism of graded $R$-modules $\varphi: R^{n+1} \rightarrow \Omega_{R / K}^{1}(1)$ with $\varphi\left(\left(F_{0}, \ldots, F_{n}\right)\right)=F_{0} d x_{0}+\cdots+F_{n} d x_{n}$ for $F_{0}, \ldots, F_{n} \in R$, and the kernel of $\varphi$ is $\mathscr{K}$. Thus the exact sequence (1) follows from Scheja and Storch (1988).

From the coordinates of points $p_{1}, \ldots, p_{s}$ of $\mathbb{X}$, we form the matrix

$$
\mathscr{A}_{\mathbb{X}}=\left(p_{i j}\right)_{\substack{i=1, \ldots, s \\ j=0, \ldots, n}} \in \operatorname{Mat}_{s \times(n+1)}(K),
$$

and we let $\rho_{\mathbb{X}}$ denote the rank of the matrix $\mathscr{A}_{\mathbb{X}}$. The next theorem shows how the rank $\rho_{\mathbb{X}}$ can be detected via the Hilbert functions of the Kähler differential modules for $\mathbb{X}$.

Theorem 2.6. The set $\mathbb{X}$ has $\rho_{\mathbb{X}}=m$ if and only if $\mathrm{HF}_{\Omega_{R / K}^{m}}(i) \neq 0$ for some $i$ and $\mathrm{HF}_{\Omega_{R / K}^{m+1}}(i)=$ 0 for all $i \in \mathbb{Z}$. 
In order to prove Theorem 2.6, we need only prove the following two lemmas.

Lemma 2.7. If $m>\rho_{\mathbb{X}}$, then $\mathrm{HF}_{\Omega_{R / K}^{m}}(i)=0$ for all $i \in \mathbb{Z}$.

Proof.

Set $M=\mathscr{K} \wedge_{R} \bigwedge_{R}^{\rho_{\mathbb{X}}}\left(R^{n+1}\right)$ and $N=\bigwedge_{R}^{\rho_{\mathbb{X}}+1}\left(R^{n+1}\right)$. According to Lemma 2.5, it suffices to show that $\operatorname{HF}_{M}(i)=\operatorname{HF}_{N}(i)$ for all $i \geq 0$. Let $\left\{e_{1}, \ldots, e_{n+1}\right\}$ be a standard basis of the graded-free $R$-module $R^{n+1}$, and let $V$ be the space of solutions of the homogeneous system of linear equations with coefficient matrix $\mathscr{A}_{\mathbb{X}}$. Then we have $t:=$ $\operatorname{dim}_{K}(V)=n+1-\rho_{\mathbb{X}}$. Let $\left\{v_{1}, \ldots, v_{t}\right\}$ be a $K$-basis of $V$. Without loss of generality, we may assume that $v_{j}=e_{j}+\sum_{k=t+1}^{n+1} a_{j k} e_{k}$ for $j=1, \ldots, t$. For each $j \in\{1, \ldots, t\}$ we see that $X_{j-1}+\sum_{k=t+1}^{n+1} a_{j k} X_{k-1} \in I_{\mathbb{X}}$, and hence $v_{j} \in \mathscr{K}$. This implies $\left\langle v_{1}, \ldots, v_{t}\right\rangle_{R} \subseteq \mathscr{K} \subseteq R^{n+1}$. have

Now we need only prove that $M^{\prime}:=\left\langle v_{1}, \ldots, v_{t}\right\rangle_{R} \wedge_{R} \wedge_{R}^{\rho_{\mathbb{X}}}\left(R^{n+1}\right)=N$. In $M^{\prime}$, we

$$
v_{j} \wedge e_{t+1} \wedge \cdots \wedge e_{n+1}=e_{j} \wedge e_{t+1} \wedge \cdots \wedge e_{n+1} .
$$

If we can show that $e_{i_{1}} \wedge \cdots \wedge e_{i_{\rho_{X}+1}} \in M^{\prime}$ for all $\left\{i_{1}, \ldots, i_{k}\right\} \subseteq\{1, \ldots, t\}$, then $\left\{i_{k+1}, \ldots, i_{\rho_{\mathbb{X}}+1}\right\} \subseteq\{t+1, \ldots, n+1\}$ and $1 \leq k \leq \rho_{\mathbb{X}}$ imply $e_{j_{1}} \wedge \cdots \wedge e_{j_{\rho_{X}+1}} \in M^{\prime}$ for all $\left\{j_{1}, \ldots, j_{k+1}\right\} \subseteq\{1, \ldots, t\}$ and $\left\{j_{k+2}, \ldots, j_{\rho_{\mathbb{X}}+1}\right\} \subseteq\{t+1, \ldots, n+1\}$. Then the set

$$
\left\{e_{i_{1}} \wedge \cdots \wedge e_{i_{\rho_{\mathbb{X}}+1}} \mid\left\{i_{1}, \ldots, i_{\rho_{\mathbb{X}}+1}\right\} \subseteq\{1, \ldots, n+1\}, \exists i_{k} \in\{1, \ldots, t\}\right\}
$$

is contained in $M^{\prime}$, and hence $M^{\prime}=N$, since this set generates $N$ as an $R$-module.

To see that $e_{j_{1}} \wedge \cdots \wedge e_{j_{\rho_{\mathbb{X}}+1}} \in M^{\prime}$, we represent

$$
\begin{aligned}
e_{j_{1}} \wedge \cdots \wedge e_{j_{\rho_{\mathbb{X}}+1}}= & \left(e_{j_{1}}+\sum_{\ell=t+1}^{n+1} a_{j_{1} \ell} e_{\ell}\right) \wedge e_{j_{2}} \wedge \cdots \wedge e_{j_{\rho_{\mathbb{X}}+1}} \\
& -\sum_{\ell=t+1}^{n+1}(-1)^{\rho_{\mathbb{X}}} a_{j_{1} \ell}\left(e_{j_{2}} \wedge \cdots \wedge e_{\left.j_{\rho_{\mathbb{X}}+1} \wedge e_{\ell}\right)}\right. \\
= & v_{j_{1}} \wedge e_{j_{2}} \wedge \cdots \wedge e_{j_{\rho_{\mathbb{X}}+1}} \\
& -\sum_{\ell=t+1}^{n+1}(-1)^{\rho_{\mathbb{X}}} a_{j_{1} \ell}\left(e_{j_{2}} \wedge \cdots \wedge e_{j_{k+1}}\right) \wedge\left(e_{j_{k+2}} \wedge \cdots \wedge e_{j_{\rho_{\mathbb{X}}+1}} \wedge e_{\ell}\right) .
\end{aligned}
$$

It follows from the hypothesis that $e_{j_{1}} \wedge \cdots \wedge e_{j_{\rho_{\mathbb{X}}+1}} \in M^{\prime}$, as desired.

Lemma 2.8. We have $\mathrm{HF}_{\Omega_{R / K}^{\rho_{X}}}(i) \neq 0$ for some $i \in \mathbb{Z}$.

Proof.

Using the notation given in the proof of Lemma 2.7, let $U=\left\langle e_{t+1}, \ldots, e_{n+1}\right\rangle_{R}$, $V=\left\langle v_{1}, \ldots, v_{t}\right\rangle_{R}$, and $N=\bigwedge_{R}^{\rho_{\mathbb{X}}}\left(R^{n+1}\right)$. Then $U, V$ are graded-free $R$-modules of rank $\rho_{\mathbb{X}}$ and $t$, respectively. We want to show that $V \wedge_{R} \bigwedge_{R}^{\rho_{\mathbb{X}}-1}\left(R^{n+1}\right) \subsetneq N$. We have $V \oplus U=R^{n+1}$ 
and

$$
\begin{aligned}
V \wedge_{R} \bigwedge_{R}^{\rho_{\mathbb{X}}-1}\left(R^{n+1}\right) & =V \wedge_{R} \bigwedge_{R}^{\rho_{\mathbb{X}}-1}(V \oplus U) \\
& =V \wedge_{R}\left(\sum_{i=0}^{\rho_{\mathbb{X}}-1} \bigwedge_{R}^{\rho_{\mathbb{X}}-1-i}(V) \otimes_{R} \bigwedge_{R}^{i}(U)\right) \\
& =\sum_{i=0}^{\rho_{\mathbb{X}}-1} \bigwedge_{R}^{\rho_{\mathbb{X}}-i}(V) \otimes_{R} \bigwedge_{R}^{i}(U),
\end{aligned}
$$

where the second equality follows from Lemma 2.4. Since $U \cap V=\langle 0\rangle$, the graded-free $R$-module $V \wedge_{R} \wedge_{R}^{\rho_{\mathbb{X}}-1}\left(R^{n+1}\right)$ has

$$
\operatorname{rank}\left(V \wedge_{R} \bigwedge_{R}^{\rho_{\mathbb{X}}-1}\left(R^{n+1}\right)\right)=\sum_{i=0}^{\rho_{\mathbb{X}}-1}\left(\begin{array}{c}
n+1-\rho_{\mathbb{X}} \\
\rho_{\mathbb{X}}-i
\end{array}\right) \cdot\left(\begin{array}{c}
\rho_{\mathbb{X}} \\
i
\end{array}\right)
$$

$N$ is a graded-free $R$-module of $\operatorname{rank}(N)=\sum_{i=0}^{\rho_{\mathbb{X}}}\left(\begin{array}{c}n+1-\rho_{\mathbb{X}} \\ \rho_{\mathbb{X}}-i\end{array}\right) \cdot\left(\begin{array}{c}\rho_{\mathbb{X}} \\ i\end{array}\right)$, and consequently, $\operatorname{rank}(N)-$ $\operatorname{rank}\left(V \wedge_{R} \bigwedge_{R}^{\rho_{\mathbb{X}}-1}\left(R^{n+1}\right)\right)=1$ or $V \wedge_{R} \bigwedge_{R}^{\rho_{\mathbb{X}}-1}\left(R^{n+1}\right) \subsetneq N$. An application of the exact sequence (1) with $V \subseteq \mathscr{K}$ yields $\mathrm{HF}_{\Omega_{R / K}^{\rho_{\mathbb{X}}}}(i) \neq 0$ for some $i \in \mathbb{Z}$.

Due to Lemmas 2.7 and 2.8, $\mathrm{HF}_{\Omega_{R / K}^{m}}(i) \neq 0$ for some $i$ and $\mathrm{HF}_{\Omega_{R / K}^{m+1}}(i)=0$ for all $i \in \mathbb{Z}$ if and only if $\rho_{\mathbb{X}}=m$. Thus, Proposition 2.6 is completely proved.

Now let us look at an explicit example.

Example 2.9. Let $\mathbb{X}=\left\{p_{1}, \ldots, p_{6}\right\} \subseteq \mathbb{P}^{4}$ be the set of six $\mathbb{Q}$-rational points, where $p_{1}=$ $(1: 0: 0: 0: 1), p_{2}=(1:-1: 1:-1: 2), p_{3}=(1: 1: 1: 1: 2), p_{4}=(1: 2: 4: 8:-1)$, $p_{5}=(1:-2: 4:-8: 11)$, and $p_{6}=(1: 3: 9: 27:-14)$ are on the intersection of a twisted cubic curve defined by $X_{0} X_{2}-X_{1}^{2}, X_{1} X_{3}-X_{2}^{2}, X_{0} X_{3}-X_{1} X_{2}$ and a hyperplane defined by $F_{1}=X_{0}+X_{1}+X_{2}-X_{3}-X_{4}$.

By forming $\mathscr{A}_{\mathbb{X}}$ and calculating its rank, we have $\rho_{\mathbb{X}}=4$. The homogeneous vanishing ideal $I_{\mathbb{X}}$ of $\mathbb{X}$ is generated by $\left\{F_{1}, F_{2}, F_{3}, F_{4}, F_{5}\right\}$ with

$$
\begin{aligned}
& F_{2}=X_{1}^{2}-2 X_{2} X_{3}+X_{3}^{2}-X_{2} X_{4}+X_{3} X_{4}, \\
& F_{3}=X_{1} X_{2}+\frac{23}{22} X_{2} X_{3}-\frac{8}{11} X_{3}^{2}-\frac{3}{11} X_{1} X_{4}+\frac{4}{11} X_{2} X_{4}-\frac{3}{4} X_{3} X_{4}, \\
& F_{4}=X_{2}^{2}-\frac{1}{22} X_{2} X_{3}-\frac{3}{11} X_{3}^{2}+\frac{3}{11} X_{1} X_{4}-\frac{4}{11} X_{2} X_{4}-\frac{1}{4} X_{3} X_{4}, \\
& F_{5}=X_{1} X_{3}-\frac{1}{22} X_{2} X_{3}-\frac{3}{11} X_{3}^{2}+\frac{3}{11} X_{1} X_{4}-\frac{4}{11} X_{2} X_{4}-\frac{1}{4} X_{3} X_{4}
\end{aligned}
$$

and $\mathrm{HF}_{\mathbb{X}}=\mathrm{HF}_{R}: 1466 \cdots$. In this case, we also have $\mathrm{HF}_{\Omega_{R / K}^{4}}(4)=1 \neq 0$ and $\mathrm{HF}_{\Omega_{R / K}^{5}}(i)=0$ for all $i \in \mathbb{Z}$.

Remark 2.10. When $\mathbb{X}=\left\{p_{1}, \ldots, p_{s}\right\} \subset \mathbb{P}^{n}$ is a set of $s$ distinct $K$-rational points that lie 
on a line, the Hilbert functions of $\mathbb{X}$ and of $\Omega_{R_{\mathbb{X}} / K}^{m}$ satisfy

$$
\begin{aligned}
& \mathrm{HF}_{\mathbb{X}}: \quad 1234 \cdots s-1 s s \cdots \\
& \mathrm{HF}_{\Omega_{R_{\mathbb{X}} / K}^{1}}: 0246 \cdots(2 s-2)(2 s-1)(2 s-2)(2 s-3) \cdots(s+1) s s \cdots \\
& \mathrm{HF}_{\Omega_{R_{\mathbb{X}}^{2} / K}^{2}}: \quad 00012 \cdots(s-2)(s-1)(s-2) \cdots 2100 \cdots
\end{aligned}
$$

and $\mathrm{HF}_{\Omega_{R_{\mathbb{X}} / K}^{m}}(i)=0$ for $m \geq 3$ and for all $i \in \mathbb{Z}$.

We end this section with a relation between the Hilbert functions of the Kähler differential modules for $\mathbb{X}$ and its subsets. This property is also true when $\mathbb{X}$ is a 0 dimensional scheme in $\mathbb{P}^{n}$.

Lemma 2.11. Let $\mathbb{Y}$ be a subset of $\mathbb{X}$ and let $R_{\mathbb{Y}}$ be the homogeneous coordinate of $\mathbb{Y}$. Then $1 \leq m \leq n+1$ and for all $i \in \mathbb{Z}$ we have

$$
\mathrm{HF}_{\Omega_{R_{\mathbb{Y}} / K}^{m}}(i) \leq \mathrm{HF}_{\Omega_{R / K}^{m}}(i)
$$

Proof.

We know that $I_{\mathbb{X}} \subseteq I_{\mathbb{Y}}$, and so there is a surjective homomorphism of rings $\pi$ : $R \rightarrow R_{\mathbb{Y}}=S / I_{\mathbb{Y}}$. This map induces a surjective homomorphism of graded $R$-modules $\phi: \Omega_{R / K}^{1} \rightarrow \Omega_{R_{\mathbb{Y}} / K}^{1}$. For $1 \leq m \leq n+1$, the map $\phi$ induces a surjective homomorphism of graded $R$-modules $\phi^{(m)}: \Omega_{R / K}^{m} \rightarrow \Omega_{R_{\mathbb{Y}} / K}^{m}$. Therefore we get the inequality $\mathrm{HF}_{\Omega_{R_{\mathbb{Y}} / K}^{m}}(i) \leq$ $\mathrm{HF}_{\Omega_{R / K}^{m}}(i)$ for all $i \in \mathbb{Z}$.

\section{SEVERAL CONFIGURATIONS OF POINTS IN THE PROJECTIVE PLANE}

In this section, we restrict our attention to the sets of $K$-rational points $\mathbb{X}=\left\{p_{1}, \ldots\right.$, $\left.p_{s}\right\}$ in the projective plane $\mathbb{P}^{2}$. More precisely, we will discuss some geometrical configurations of points $\mathbb{X}$ in $\mathbb{P}^{2}$ which are reflected in terms of the Hilbert function of the module of Kähler differential 3-forms. Firstly, we look at a representation of this module. We denote

$$
\partial I_{\mathbb{X}}:=\left\langle\frac{\partial F}{\partial X_{i}} \mid F \in I_{\mathbb{X}}, 0 \leq i \leq n\right\rangle \subseteq S .
$$

The ideal $\partial I_{\mathbb{X}}$ is also known as the $n$-th Jacobian ideal (or the $n$-th Kähler different) of $R / K$ (see, e.g., Kunz, 1986, Section 10). We can use this notation to give an explicit description of the module of Kähler differential 3-forms of $R / K$ as follows.

Lemma 3.1. There is an isomorphism of graded $R$-modules

$$
\Omega_{R / K}^{3} \cong\left(S / \partial I_{\mathbb{X}}\right)(-n-1) .
$$

In particular, $\mathrm{HF}_{\Omega_{R / K}^{3}}(i)=\mathrm{HF}_{S / \partial I_{\mathbb{X}}}(i-n-1)$ for all $i \in \mathbb{Z}$. 
Proof.

See Kreuzer et al. (2019), Corollary 2.3.

Now we may give a criterion for the set $\mathbb{X}$ to lie on a conic $\mathscr{C}=\mathscr{Z}^{+}(C)$, where

$$
C=\sum_{0 \leq i, j \leq 2} a_{i j} X_{i} X_{j} \quad\left(a_{i j} \in K\right)
$$

Proposition 3.2. A set of $s$ distinct $K$-rational points $\mathbb{X} \subseteq \mathbb{P}^{2}$ lies on a conic $\mathscr{C}=\mathscr{Z}^{+}(C)$ that is not a double line if and only if we have $\mathrm{HF}_{\Omega_{R / K}^{3}}(i) \leq 1$ for all $i \in \mathbb{Z}, \mathrm{HF}_{\Omega_{R / K}^{3}}(3)=1$.

Proof.

We may assume that $C \neq a \ell^{2}$ for any linear form $\ell \in S$ and $a \in K$ and that $a_{00} \neq 0$ (after a change of coordinates). We have $\frac{\partial C}{\partial X_{i}}=\sum_{j=0}^{2} a_{i j} X_{j} \in \partial I_{\mathbb{X}}$ for $i=0,1,2$, as $C \in I_{\mathbb{X}}$. Put $\mathscr{A}:=\left(a_{i j}\right)_{i, j=0,1,2} \in \operatorname{Mat}_{3}(K)$. Then $\operatorname{rank}(\mathscr{A})=1$ if and only if $\mathscr{A}$ is of the form

$$
\mathscr{A}=\left(\begin{array}{ccc}
a_{00} & a_{01} & a_{02} \\
a_{01} & \frac{a_{01}^{2}}{a_{00}} & \frac{a_{01} a_{02}}{a_{00}} \\
a_{02} & \frac{a_{01} a_{02}}{a_{00}} & \frac{a_{02}^{2}}{a_{00}}
\end{array}\right) .
$$

In this case we get $C=\frac{1}{a_{00}} \ell^{2}$ with $\ell=\frac{\partial C}{\partial X_{0}}=\sum_{j=0}^{2} a_{0 j} X_{j}$, which is impossible. Hence, $\operatorname{rank}(\mathscr{A}) \geq 2$, and suppose that the two first rows of $\mathscr{A}$ are linearly independent. Then $\frac{\partial C}{\partial X_{0}}, \frac{\partial C}{\partial X_{1}}$ form a regular sequence for $S$. Notice that

$$
J:=\left\langle\frac{\partial C}{\partial X_{0}}, \frac{\partial C}{\partial X_{1}}\right\rangle_{S} \subseteq\left\langle\frac{\partial C}{\partial X_{i}} \mid 0 \leq i \leq 2\right\rangle \subseteq \partial I_{\mathbb{X}}
$$

Hence, according to Lemma 3.1, for all $i \in \mathbb{Z}$ we have

$$
\mathrm{HF}_{\Omega_{R / K}^{3}}(i)=\mathrm{HF}_{S / \partial I_{\mathbb{X}}}(i-3) \leq \mathrm{HF}_{S / J}(i-3) \leq 1
$$

On the other hand, we also have $\partial I_{\mathbb{X}} \subseteq\left\langle X_{0}, X_{1}, X_{2}\right\rangle_{S}$ because $\mathbb{X}$ does not lie on a line. This implies $\mathrm{HF}_{\Omega_{R / K}^{3}}(3)=1$.

Conversely, assume that $\mathrm{HF}_{\Omega_{R / K}^{3}}(i) \leq 1$ for all $i \in \mathbb{Z}$ and $\mathrm{HF}_{\Omega_{R / K}^{3}}(3)=1$. Theorem 2.6 guarantees that $\mathbb{X}$ lies neither on a line nor on a double line. If $\mathbb{X}$ does not lie on any conic, then the homogeneous vanishing ideal $I_{\mathbb{X}}$ is generated in degrees greater than 2, and hence $\mathrm{HF}_{\partial I_{\mathbb{X}}}(1)=0$. By Lemma 3.1, we get $\mathrm{HF}_{\Omega_{R / K}^{3}}(4)=3>1$, a contradiction. Therefore $\mathbb{X}$ must lie on a conic. for all $i \in \mathbb{N}$.

Due to the proof of Proposition 3.2, $\mathbb{X}$ lies on a line if and only if $\mathrm{HF}_{\Omega_{R / K}^{3}}(i)=0$

Lemma 3.3. If the set $\mathbb{X}=\left\{p_{1}, \ldots, p_{s}\right\} \subseteq \mathbb{P}^{2}$ lies on a non-singular conic $\mathscr{C}=\mathscr{Z}^{+}(C)$, then $\mathrm{HF}_{\Omega_{R / K}^{3}}(i)=0$ for $i \neq 3$ and $\mathrm{HF}_{\Omega_{R / K}^{3}}(3)=1$. 
Proof.

Let $C$ be given in (2) and let $\mathscr{A}=\left(a_{i j}\right)_{i, j=0,1,2} \in \operatorname{Mat}_{3}(K)$ be the coefficient matrix of $C$. It is well-known that $\mathscr{C}$ is a non-singular conic if and only if $\operatorname{rank}(\mathscr{A})=3$. This last condition yields $\left\langle X_{0}, X_{1}, X_{2}\right\rangle_{S}=\left\langle\frac{\partial C}{\partial X_{i}} \mid 0 \leq i \leq 2\right\rangle_{S} \subseteq \partial I_{\mathbb{X}}$. Hence $\left\langle X_{0}, X_{1}, X_{2}\right\rangle_{S}=\partial I_{\mathbb{X}}$, and the claim follows by Lemma 3.1.

Next we consider the case that the set $\mathbb{X}$ lies on two different lines.

Proposition 3.4. Let $\mathbb{X} \subseteq \mathbb{P}^{2}$ be a set of $s$ distinct $K$-rational points that lie on two different lines.

(a) If $s=5$ and no four points of $\mathbb{X}$ lie on the same line, then

$$
\mathrm{HF}_{\Omega_{R / K}^{3}}: 0 \begin{array}{lllllllll}
0 & 0 & 1 & 1 & 0 & \cdots
\end{array}
$$

(b) If $s>5$ and there exist five points such that no four of them lie on the same line, then

$$
\mathrm{HF}_{\Omega_{R / K}^{3}}(3)=\mathrm{HF}_{\Omega_{R / K}^{3}}(4)=1
$$

Proof.

Suppose that $\mathbb{X}$ lies on two lines defined by $\ell_{1}=\sum_{j=0}^{2} a_{j} X_{j}$ and $\ell_{2}=\sum_{j=0}^{2} b_{j} X_{j}$ with $a_{i}, b_{j} \in K$. Note that $\operatorname{gcd}\left(\ell_{1}, \ell_{2}\right)=1$ and, without loss of generality, assume that $a_{0} b_{1}-a_{1} b_{0} \neq 0$. We first treat the case of five points.

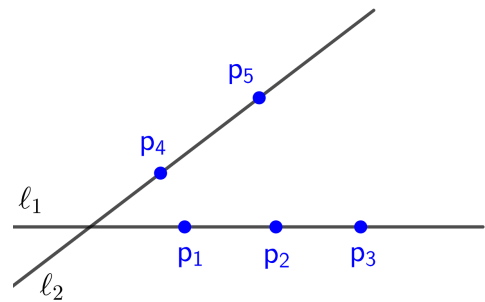

(a)

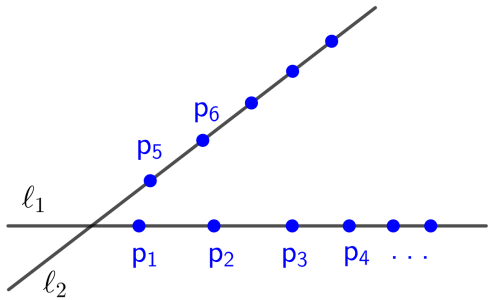

(b)

(a) Assume that $\mathbb{X}_{1}=\left\{p_{1}, p_{2}, p_{3}\right\} \subseteq \mathscr{Z}^{+}\left(\ell_{1}\right)$ and $\mathbb{X}_{2}=\left\{p_{4}, p_{5}\right\} \subseteq \mathscr{Z}^{+}\left(\ell_{2}\right)$. Then we have $I_{\mathbb{X}_{1}}=\left\langle\ell_{1}^{\prime} \ell_{2}^{\prime} \ell_{3}^{\prime}, \ell_{1}\right\rangle_{S}$ and $I_{\mathbb{X}_{2}}=\left\langle\ell_{4}^{\prime} \ell_{5}^{\prime}, \ell_{2}\right\rangle_{S}$ for suitable linear forms $\ell_{i}^{\prime}=\sum_{j=0}^{2} c_{i j} X_{j}$ $\left(i=1, \ldots, 5, c_{i j} \in K\right)$. Clearly, the initial degree of the ideal $I_{\mathbb{X}}$ is 2 and $I_{\mathbb{X}}=I_{\mathbb{X}_{1}} \cap I_{\mathbb{X}_{2}}$. For a non-zero element $F \in\left(I_{\mathbb{X}}\right)_{2}$, we may write

$$
F=F_{1} \ell_{1}=F_{2} \ell_{2}+c \ell_{4}^{\prime} \ell_{5}^{\prime}
$$

for some $c \in K$ and $F_{1}, F_{2} \in S_{1}$. Since $\ell_{1}\left(p_{4}\right) \neq 0$ and $\ell_{1}\left(p_{5}\right) \neq 0$, we have $F_{1}\left(p_{4}\right)=$ $F_{1}\left(p_{5}\right)=0$, and so $F_{1}=c^{\prime} \ell_{2}$ with $c^{\prime} \in K \backslash\{0\}$. Hence $F \in\left(I_{\mathbb{X}}\right)_{2}$ if and only if $F=\left\langle\ell_{1} \ell_{2}\right\rangle_{K}$. Observe that

$$
\frac{\partial \ell_{1} \ell_{2}}{\partial X_{i}}=\ell_{1} \frac{\partial \ell_{2}}{\partial X_{i}}+\ell_{2} \frac{\partial \ell_{1}}{\partial X_{i}}=b_{i} \ell_{1}+a_{i} \ell_{2} \in \partial I_{\mathbb{X}} \quad(i=0,1,2)
$$

and $\left\langle b_{0} \ell_{1}+a_{0} \ell_{2}, b_{1} \ell_{1}+a_{1} \ell_{2}\right\rangle_{K}=\left\langle\ell_{1}, \ell_{2}\right\rangle_{K}$, as $a_{0} b_{1}-a_{1} b_{0} \neq 0$. Since $b_{2} \ell_{1}+a_{2} \ell_{2} \in$ $\left\langle\ell_{1}, \ell_{2}\right\rangle_{K}$, we obtain $\left\langle\ell_{1}, \ell_{2}\right\rangle_{K}=\left(\partial I_{\mathbb{X}}\right)_{1}$. This implies

$$
\mathrm{HF}_{\Omega_{R / K}^{3}}(4)=\operatorname{HF}_{S / \partial I_{\mathbb{X}}}(1)=3-\operatorname{dim}_{K}\left(\left\langle\ell_{1}, \ell_{2}\right\rangle_{K}\right)=1 .
$$


Furthermore, we have $\ell_{1} \ell_{4}^{\prime} \ell_{5}^{\prime} \in I_{\mathbb{X}}$, and hence, for $a_{i} \neq 0$, we get

$$
\ell_{4}^{\prime} \ell_{5}^{\prime}=\frac{1}{a_{i}}\left(\frac{\partial \ell_{1} \ell_{4}^{\prime} \ell_{5}^{\prime}}{\partial X_{i}}-\ell_{1}\left(\frac{\partial \ell_{4}^{\prime} \ell_{5}^{\prime}}{\partial X_{i}}\right)\right) \in \partial I_{\mathbb{X}} .
$$

In particular, $\left\langle\ell_{1}, \ell_{2}, \ell_{4}^{\prime} \ell_{5}^{\prime}\right\rangle_{S} \subseteq \partial I_{\mathbb{X}}$. If $\ell_{4}^{\prime} \ell_{5}^{\prime}=H_{1} \ell_{1}+H_{2} \ell_{2}$ for some $H_{1}, H_{2} \in S_{1}$, then $H_{1}\left(p_{4}\right)=H_{1}\left(p_{5}\right)=0$ or $H_{1}=c^{\prime \prime} \ell_{2}$, and thus $\ell_{2} \mid \ell_{4}^{\prime} \ell_{5}^{\prime}$, but this is impossible. Hence $\ell_{4}^{\prime} \ell_{5}^{\prime} \notin\left\langle\ell_{1}, \ell_{2}\right\rangle_{S}$. It follows that

$$
\operatorname{dim}_{K}\left(\left\langle\ell_{1}, \ell_{2}, \ell_{4}^{\prime} \ell_{5}^{\prime}\right\rangle_{S}\right)_{2}=\operatorname{dim}_{K}\left(\partial I_{\mathbb{X}}\right)_{2}=\operatorname{dim}_{K}\left(S_{2}\right)=6,
$$

and consequently, $\left\langle\ell_{1}, \ell_{2}, \ell_{4}^{\prime} \ell_{5}^{\prime}\right\rangle_{S}=\partial I_{\mathbb{X}}$ and $\operatorname{HF}_{\partial I_{\mathbb{X}}}(i)=\operatorname{HF}_{S}(i)$ for all $i \geq 2$. Therefore, $\mathrm{HF}_{\Omega_{R / K}^{3}}(i)=0$ for all $i>4$.

(b) Because the set $\mathbb{X}$ lies on two different lines, Proposition 3.2 implies that $\mathrm{HF}_{\Omega_{R / K}^{3}}(3)=1$ and $\mathrm{HF}_{\Omega_{R / K}^{3}}(4) \leq 1$. Let $\mathbb{Y}$ be the set of five points of $\mathbb{X}$ such that no four points of $\mathbb{Y}$ lie on the same line. By (a) and Lemma 2.11, we have $1=\mathrm{HF}_{\Omega_{R_{\mathbb{Y}} / K}^{3}}(4) \leq$ $\mathrm{HF}_{\Omega_{R / K}^{3}}(4) \leq 1$, and hence the last inequality becomes an equality.

When there is a line passing through all but one point of $\mathbb{X}$, we have the following property.

Lemma 3.5. Let $\mathbb{X}=\mathbb{Y} \cup\left\{p_{s}\right\} \subseteq \mathbb{P}^{2}$, where $\mathbb{Y}=\left\{p_{1}, \ldots, p_{s-1}\right\}$ is a set of $s-1$ distinct $K$-rational points on a line $\mathscr{Z}^{+}(\ell)$ and $p_{s} \notin \mathscr{Z}^{+}(\ell)$. Then

$$
\mathrm{HF}_{\Omega_{R / K}^{3}}(3)=1 \quad \text { and } \quad \mathrm{HF}_{\Omega_{R / K}^{3}}(i)=0, \quad \forall i \neq 3 .
$$

Proof.

Since $I_{\mathbb{Y}}=\bigcap_{j=1}^{s-1} \mathfrak{p}_{j}$, if $\mathfrak{p}_{j}=\left\langle\ell, \ell_{j}\right\rangle_{S}$ with a linear form $\ell_{j} \in S$ for $j=1, \ldots, s-1$, then $I_{\mathbb{Y}}$ is generated by $\ell$ and $F=\prod_{j=1}^{s-1} \ell_{j}$. Let us write $\mathfrak{p}_{s}=\left\langle L_{1}, L_{2}\right\rangle_{S}$ with suitable $L_{1}=\sum_{j=0}^{2} a_{i} X_{i}, L_{2}=\sum_{j=0}^{2} b_{i} X_{i} \in S_{1}$ such that $p_{1} \in \mathscr{Z}^{+}\left(L_{1}\right)$ and $p_{2} \in \mathscr{Z}^{+}\left(L_{2}\right)$. Such $L_{1}, L_{2} \in S_{1}$ exist, since $p_{s} \notin \mathscr{Z}^{+}(\ell)$. Then we have $I_{\mathbb{X}}=\bigcap_{j=1}^{s} \mathfrak{p}_{j}=\langle\ell, F\rangle_{S} \cap\left\langle L_{1}, L_{2}\right\rangle_{S}$.

We claim that $\operatorname{dim}_{K}\left(\left\langle\ell, L_{1}, L_{2}\right\rangle_{K}\right)=3$. If there are $a, b, c \in K$ such that $a \ell+b L_{1}+$ $c L_{2}=0$, then $0=a \ell\left(p_{1}\right)+b L_{1}\left(p_{1}\right)+c L_{2}\left(p_{1}\right)=c L_{2}\left(p_{1}\right)$ and $0=a \ell\left(p_{2}\right)+b L_{1}\left(p_{2}\right)+$ $c L_{2}\left(p_{2}\right)=b L_{1}\left(p_{2}\right)$, and so $b=c=0$ and $a \ell=0$. This means that $a=b=c=0$ and the claim follows.

Consequently, we get $\left\langle\ell, L_{1}, L_{2}\right\rangle_{K}=\left\langle X_{0}, X_{1}, X_{2}\right\rangle_{K}$. Let $\ell=\sum_{j=0}^{2} c_{i} X_{i}$ with $c_{i} \in$ $K$ and suppose without loss of generality that $a_{0} c_{1}-a_{1} c_{0} \neq 0$ and $b_{0} c_{1}-b_{1} c_{0} \neq 0$ (as $\left\{\ell, L_{1}, L_{2}\right\}$ is linearly independent over $K$ ). Since $\ell L_{1}, \ell L_{2} \in I_{\mathbb{X}}$, we have $a_{i} \ell+c_{i} L_{1}, b_{i} \ell+$ $c_{i} L_{2} \in \partial I_{\mathbb{X}}$ for every $i=0,1,2$. We have

$$
\left\langle a_{i} \ell+c_{i} L_{1}, b_{i} \ell+c_{i} L_{2} \mid i=0,1,2\right\rangle_{K}=\left\langle\ell, L_{1}, L_{2}\right\rangle_{K}=\left\langle X_{0}, X_{1}, X_{2}\right\rangle_{K},
$$

and subsequently we get $\partial I_{\mathbb{X}}=\left\langle X_{0}, X_{1}, X_{2}\right\rangle_{S}$. Therefore, Lemma 3.1 yields

$$
\mathrm{HF}_{\Omega_{R / K}^{3}}(3)=\mathrm{HF}_{S / \partial I_{\mathbb{X}}}(0)=1, \quad \mathrm{HF}_{\Omega_{R / K}^{3}}(i)=\mathrm{HF}_{S / \partial I_{\mathbb{X}}}(i-3)=0
$$

for all $i \neq 3$. 
Note that the Castelnuovo function of $\mathbb{X}$ is defined by

$$
\Delta \mathrm{HF}_{\mathbb{X}}(i):=\mathrm{HF}_{\mathbb{X}}(i)-\mathrm{HF}_{\mathbb{X}}(i-1)
$$

for all $i \in \mathbb{Z}$. In the proof of the lemma, we see that $I_{\mathbb{X}}=\langle\ell, F\rangle_{S} \cap\left\langle L_{1}, L_{2}\right\rangle_{S} \supseteq\left\langle\ell L_{1}, \ell L_{2}, F L_{1}\right.$, $\left.F L_{2}\right\rangle_{S}$. It follows that $\Delta \mathrm{HF}_{\mathbb{X}}(2)=1$, and therefore $\Delta \mathrm{HF}_{\mathbb{X}}(i) \leq 1$ for all $i \geq 2$ by Kreuzer and Robbiano (2005), Corollary 5.5.28. This proves the following corollary.

Corollary 3.6. In the setting of Lemma 3.5, we have $\Delta \mathrm{HF}_{\mathbb{X}}(i) \leq 1$ for all $i \geq 2$.

Using Bézout's theorem (see, e.g., Ueno, 1999, Theorem 1.32), we get the following consequence for a set of points on a non-singular conic in $\mathbb{P}^{2}$.

Corollary 3.7. If $\mathbb{X}$ is a set of $s \geq 5$ distinct $K$-rational points in $\mathbb{P}^{2}$ that are on a nonsingular conic $\mathscr{C}=\mathscr{Z}^{+}(C)$, then $\Delta \mathrm{HF}_{\mathbb{X}}(2)=2$.

Proof.

By Bézout's theorem and $s \geq 5, I_{\mathbb{X}}$ has only one non-zero homogeneous polynomial $F$ of degree 2. In this case we must have $F=a C$ with some $a \in K \backslash\{0\}$. Let $\left\{C, F_{1}, \ldots, F_{t}\right\}, \operatorname{deg}\left(F_{i}\right)>2$, be a homogeneous system of generators of $I_{\mathbb{X}}$. Then the Hilbert function of $\mathbb{X}$ is given by

$$
\mathrm{HF}_{\mathbb{X}}: 135 * * \cdots
$$

In particular, we obtain $\Delta \mathrm{HF}_{\mathbb{X}}(2)=2$.

Combining the above results we get the following classification of sets of points corresponding to values of the Hilbert function of the module of Kähler differentials.

Theorem 3.8. Let $\mathbb{X}$ be a set of $s \geq 5$ distinct $K$-rational points in $\mathbb{P}^{2}$.

(a) $\mathbb{X}$ lies on two different lines and no $s-1$ points of $\mathbb{X}$ lie on the same line, when $\mathrm{HF}_{\Omega_{R / K}^{3}}(3)=1$ and $\mathrm{HF}_{\Omega_{R / K}^{3}}(4)=1$.

(b) If $\mathrm{HF}_{\Omega_{R / K}^{3}}(3)=1$ and $\mathrm{HF}_{\Omega_{R / K}^{3}}(i)=0$ for $i \neq 3$, then $\mathbb{X}$ either contains $s-1$ points on the same line or lies on a non-singular conic depending on whether the value of $\Delta \mathrm{HF}_{\mathbb{X}}(2)$ equals 1 or not.

Proof.

We see that $\mathbb{X}$ lies on a conic if and only if the Hilbert function of $\Omega_{R / K}^{3}$ satisfies $\mathrm{HF}_{\Omega_{R / K}^{3}}(4) \leq 1$ and $\mathrm{HF}_{\Omega_{R / K}^{3}}(3)=1$ by Theorem 2.6 and Proposition 3.2. We consider the following three possible configurations of the points of $\mathbb{X}$.

Case 1: If $\mathbb{X}$ lies on two different lines, such that no $s-1$ points lie on the same line, then Proposition 3.4 shows that $\mathrm{HF}_{\Omega_{R / K}^{3}}(3)=\mathrm{HF}_{\Omega_{R / K}^{3}}(4)=1$.

Case 2: In the case that there is a single line passing through all but one point of $\mathbb{X}$, by Lemma 3.5 we have $\mathrm{HF}_{\Omega_{R / K}^{3}}(3)=1$ and $\mathrm{HF}_{\Omega_{R / K}^{3}}(4)=0$. Furthermore, Corollary 3.6 shows that $\Delta \mathrm{HF}_{\mathbb{X}}(2)=1$. 
Case 3: When $\mathbb{X}$ lies on a non-singular conic, Lemma 3.3 implies $\operatorname{HF}_{\Omega_{R / K}^{3}}(3)=1$ and $\mathrm{HF}_{\Omega_{R / K}^{3}}(4)=0$. In this case we have $\Delta \mathrm{HF}_{\mathbb{X}}(2)=2$ by Corollary 3.7.

Altogether, the theorem is completely proved.

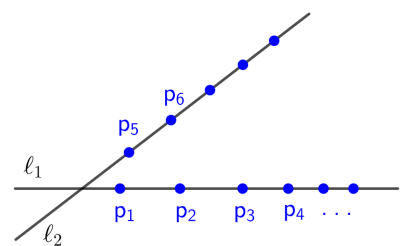

$\left(c_{1}\right)$

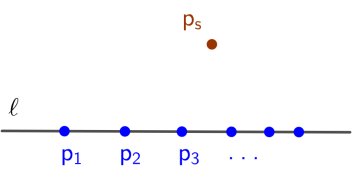

$\left(c_{2}\right)$

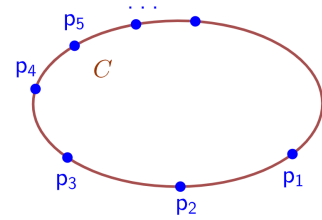

$\left(c_{3}\right)$

The following example shows us how to distinguish two sets of $K$-rational points with the same cardinality using the modules of Kähler differentials.

Example 3.9. Let $\mathbb{X} \subseteq \mathbb{P}^{2}$ be a set of six $K$-rational points on the conic $X_{0}^{2}+2 X_{1}^{2}-X_{2}^{2}$, and let $\mathbb{Y} \subseteq \mathbb{P}^{2}$ be a set of six $K$-rational points on two lines defined by $\ell_{1}=x_{1}$ and $\ell_{2}=x_{2}$. Then the Hilbert functions of $\mathbb{X}$ and $\mathbb{Y}$ are the same and given by $\mathrm{HF}_{\mathbb{X}}=\mathrm{HF}_{\mathbb{Y}}$ : $13566 \cdots$. Also, their homogeneous vanishing ideals have the same minimal graded free resolution (see Tohăneanu \& Van Tuyl, 2013, Example 4.1). However, their modules of Kähler differential $m$-forms have the following Hilbert functions:

$$
\begin{array}{ll}
\mathrm{HF}_{\Omega_{R / K}^{1}}: 0381110766 \cdots, & \mathrm{HF}_{\Omega_{R_{\mathbb{Y}} / K}^{1}}: 0381110766 \cdots, \\
\mathrm{HF}_{\Omega_{R / K}^{2}}: 0036410 \cdots, & \mathrm{HF}_{\Omega_{R_{\mathbb{Y}}^{2} / K}^{2}}: 00365100 \cdots, 0001100 \cdots . \\
\mathrm{HF}_{\Omega_{R / K}^{3}}: 000100 \cdots, & \mathrm{HF}_{\Omega_{R_{\mathbb{Y}}^{3} / K}^{3}}: 000,
\end{array}
$$

Thus, by looking at the Hilbert functions of the modules of Kähler differential $m$-forms, one can distinguish two sets $\mathbb{X}$ and $\mathbb{Y}$.

\section{ACKNOWLEDGMENTS}

This paper is dedicated to Professors Hà Huy Vui and Ta Lê Loi on the special occasion of their birthdays. The authors were supported by Hue University under grant number DHH2021-03-159.

\section{REFERENCES}

ApCoCoA Team. (2021). Apcocoa: Applied computations in commutative algebra.ApCo CoAWiki. http://apcocoa.uni-passau.de

de Dominicis, G., \& Kreuzer, M. (1999). Kähler differentials for points in Pn. Journal of Pure and Applied Algebra, 141(2), 153-173. https://doi.org/10.1016/S0022-4049 (98)00016-4 
Kreuzer, M., Linh, T. N. K., \& Long, L. N. (2015). Kähler differentials and Kähler differents for fat point schemes. Journal of Pure and Applied Algebra, 219(10), 44794509. https://doi.org/10.1016/j.jpaa.2015.02.028

Kreuzer, M., Linh, T. N. K., \& Long, L. N. (2019). Kähler differential algebras for 0-dimensional schemes. Journal of Algebra, 501, 255-284. https://doi.org/10.1016/j.jal gebra.2017.12.023

Kreuzer, M., \& Robbiano, L. (2005). Computational commutative algebra 2. Springer.

Kunz, E. (1986). Kähler differentials. Vieweg \& Teubner Verlag Wiesbaden. https://doi .org/10.1007/978-3-663-14074-0

Kunz, E. (2005). Introduction to plane algebraic curves. Birkhäuser.

Scheja, G., \& Storch, U. (1988). Lehrbuch der algebra, Unter Einschluß der linearen Algebra, Teil 2. B.G. Vieweg \& Teubner Verlag Wiesbaden. https://doi.org/10. 1007/978-3-322-80092-3

Tohăneanu, Ş. O., \& Van Tuyl, A. (2013). Bounding invariants of fat points using a coding theory construction. Journal of Pure and Applied Algebra, 217(2), 269-279. https ://doi.org/10.1016/j.jpaa.2012.06.004

Tran, N. K. L. (2015). Kähler differential modules for 0-dimensional schemes and applications [Doctoral dissertation, University of Passau, Germany].

Ueno, K. (1999). Algebraic geometry 1: From algebraic varieties to schemes. American Mathematical Society. https://doi.org/10.1090/mmono/185 